\title{
A Practical Utility and Benefit of Pharmacogenetic-based Antidepressant Treatment Strategy for Major Depressive Disorder Patients with Difficult-to-treat
}

\author{
Kyung Ho Lee ${ }^{1}$, Won-Myong Bahk ${ }^{2}$, Soo-Jung Lee ${ }^{2}$, Alessandro Serretti ${ }^{3}$, Chi-Un Pae ${ }^{2,4}$ \\ Departments of ${ }^{1}$ Dermatology and ${ }^{2}$ Psychiatry, College of Medicine, The Catholic University of Korea, Seoul, Korea, ${ }^{3}$ Department of Biomedical \\ and Neuromotor Sciences, Psychiatric Section, University of Bologna, Bologna, Italy, ${ }^{4}$ Cell Death Disease Research Center, College of Medicine, \\ The Catholic University of Korea, Seoul, Korea
}

Objective: We report the results of pharmacogenomics-based antidepressant treatment (PGXt) results in treating treatment-resistant major depressive disorder (TRD) patients in real practice.

Methods: Nine patients were prescribed Neuropharmagen ${ }^{\mathbb{R}}$ for selection of antidepressants for individual patient and their clinical outcomes were followed.

Results: After treatment by PGXt results from current antidepressants, substantial reduction of depressive symptoms was observed at some point and maintained during observation period in six patients.

Conclusion: Our case series potentially shows the clinical utility and benefit of PGXt for treatment of TRD patients.

KEY WORDS: Treatment; Depressive disorder; Antidepressive agent; Treatment-resistance; Clinical outcome.

\section{INTRODUCTION}

The selection of antidepressant for patients with major depressive disorder (MDD) usually depends on clinical intuition, experience and preference of treating physician and thus burden from "trial-and-error" is one of barriers in fast and proper antidepressant treatment for MDD patients in routine practice. Pharmacogenomics-based antidepressant treatment (PGXt) has been recently proposed and promoted as one of available treatment options toward precision medicine in MDD [1].

According to a recent independent survey with 156 clinicians, more than $80 \%$ of participants agreed that pharmacogenomic variants may affect drug responses and have clinical utility [2]. Interestingly another survey (psychiatrists, $n=5,316$ : neurologists, $n=2,167$ ) [3] indicated the differential experience of genetic testing be-

Received: March 4, 2020 / Accepted: March 13, 2020

Address for correspondence: Chi-Un Pae

Department of Psychiatry, Bucheon St. Mary's Hospital, College

of Medicine, The Catholic University of Korea, 327 Sosa-ro,

Wonmi-gu, Bucheon 14647, Korea

E-mail: pae@catholic.ac.kr

ORCID: https://orcid.org/0000-0003-1632-4248 tween psychiatrists and neurologists despite of several common patient populations; neurologists had 5 times higher experience with genetic tests than psychiatrists in a past 6 months. The most commonly ordered categories of tests were related to stroke, neuromuscular disorders, and movement disorders for neurologists; and pharmacogenomics for psychiatrists [3].

There have been a number of clinical studies investigating PGXt effects using different methodologies [1,4-12]. According to a recent meta-analysis [13] comparing commercial PGXt kits, the pooled risk ratio for treatment response and remission of PGXt versus treatment as usual were 1.36 and 1.74, respectively, which are all in favor of PGXt. A number of PGXt tool kits have been available and studied and commercialized for routine practice, however, none of these has become common use in routine clinical practice [14].

Hence, following case series of PGXt use may enhance and promote the understanding of clinicians for practical utility and benefits of PGXt in treatment of treatment-resistant depression (TRD) patients in routine practice.

(c) This is an Open-Access article distributed under the terms of the Creative Commons Attribution Non-Commercial License (http://creativecommons.org/licenses/by-nc/4.0) which permits unrestricted non-commercial use, distribution, and reproduction in any medium, provided the original work is properly cited. 


\section{METHODS}

All data was taken from medical records.

The PGXt was prescribed by the consent of individual patient since it is not covered by government insurance in Korea, meaning that all patients were fully responsible for its payment. For brief summary of PGXt, the saliva was collected into prepared easy kit and then sent to the central lab located in Barcelona. The result was available after two to three weeks after delivery to the lab. The full results were provided on the designated website (http://international.neurofarmagen.com) upon receiving the email notice to the clinician. The final results were graphically presented with four color code as follows: 1 ) green (increased likelihood of positive response and/or lower risk of adverse drug reactions), 2) red (increased risk of adverse drug reactions), 3) yellow (need for drug dose monitoring and/or less likelihood of positive response), and 4) white (described as standard, no genetic variants relevant to the treatment have been found. use as directed). More detailed genetic information including drug-drug interaction was also provided in the final results [1].

All subjects were treated by selected antidepressants based on the results from PGXt. All the PGXt results were thoroughly provided to individual patient upon receiving the results of PGXt and patients were told about next and future treatment strategy with the use of PGXt results such as application of augmentation, combination, and switching of all current psychotropics including antidepressant. Individual patient actively participated in the treatment decision along with treating clinician.

Available clinical information including MontgomeryÅsberg Depression Rating (MADRS) was collected.

The remission was defined as having $\leq 10$ in MADRS total score at any visit point. Observation period was not specifically defined since the present study tried to find clinical outcomes in a naturalistic setting and follow-up period was quite different among all subjects.

This retrospective study was approved by institutional review board (IRB) of Bucheon St. Mary's Hospital (IRB approval number. HC20RISI0009).

\section{RESULTS}

Thirteen patients were tested by the PGXt in routine practice. Minimum follow-up time was 106 days (case 7) and the longest was 3 years (case 4). Three were diagnosed with schizophrenia and another one was a foreigner who just wanted to test his genotypic profile for comparison of current antidepressant since he has been treated with same antidepressants more than 10 year with moderate response and some adverse events. Therefore, 9 patients were included in this study. Detailed clinical information is presented in Table 1. Six patients remitted as of the last follow-up point. The mean remission time was 80.5 days after treatment of PGXt $(n=6)$, while the response time was 37.3 days $(n=6)$.

\section{Case 1}

Mr. A is a 22-year-old male college student who has been continuously depressed and treated by foreign psychiatrist while studying abroad. His first-degree relatives had suffered from MDD (attempted suicide history). Main symptoms included depressed mood, loss of interest, lack of energy, concentration difficulties, insomnia, and loss of hope, as well as fear to AEs from antidepressants. He previously tried all SSRIs without proper effects but suffered AEs including sedation, anxiety and decreased cognitive function. Based on his PGX, bupropion 300 mg/d was applied for one week, but he complained a severe headache and thus it was decreased to $150 \mathrm{mg} / \mathrm{d}$ for 7 days and increased to $300 \mathrm{mg} / \mathrm{d}$ for next 2 weeks after disappearance of headache. His depressive symptoms were remitted at day 51 ; he reported his function was completely restored and went back to foreign country to continue his study.

\section{Case 2}

Mrs. B is a 44-year-old female working as a teacher. Her symptoms included depressed mood, frequent tiredness, lack of energy, decreased concentration, emotional fluctuation, sleep disturbance, and anxiety. She was also sensitive to AEs of previous antidepressants and suffered lack of treatment response. As seen in Table 1, she had failed to show proper response from previous antidepressant treatment. After switching to escitalopram, her long-standing $\mathrm{AE}$, mild hand tremor and dry mouth substantially reduced and remission was observed at 94 days of PGXt maintaining till today.

\section{Case 3}

Mrs. C is a 43-year-old female, a health professional. 


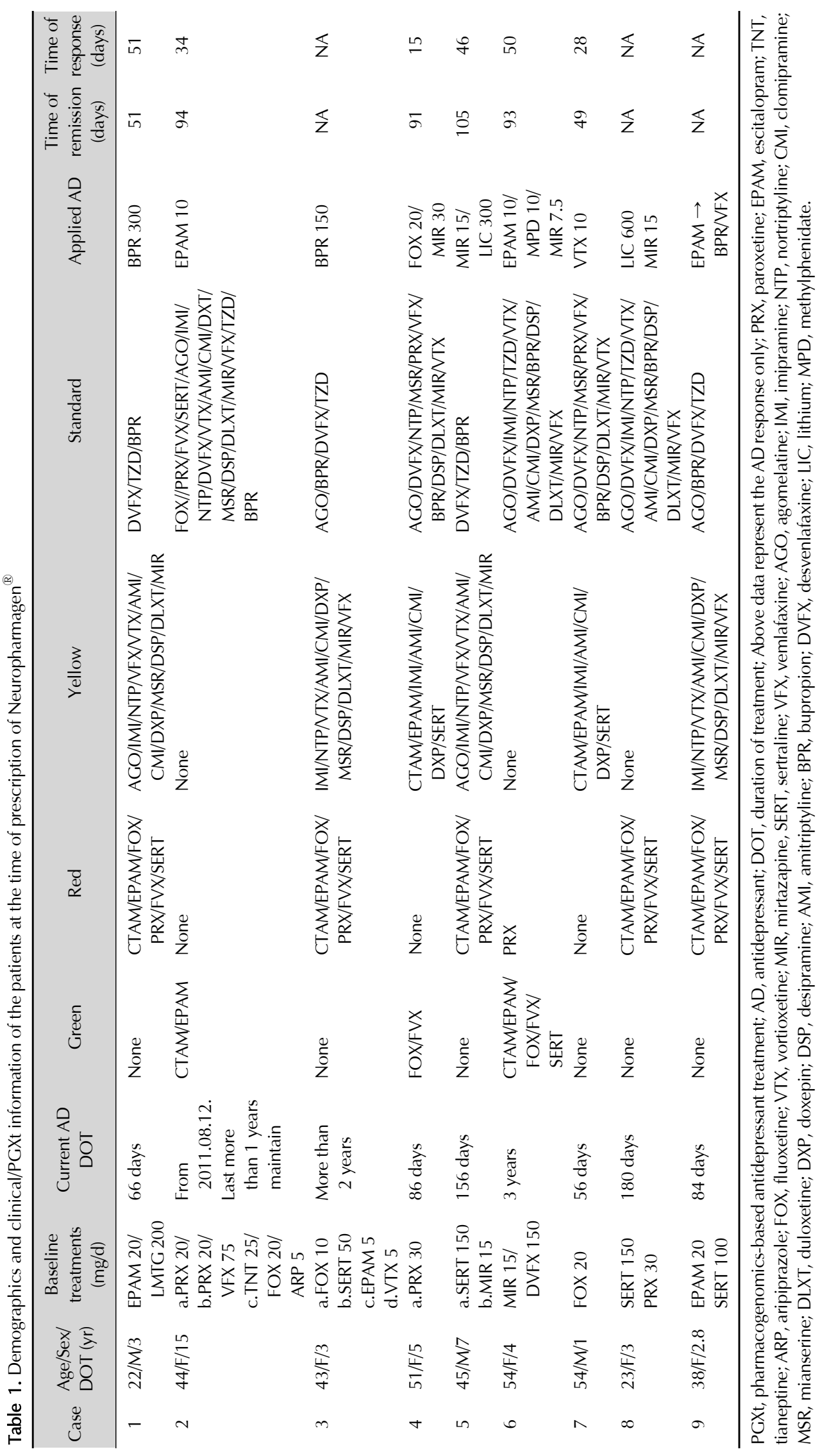


Her symptoms included depressed mood, sadness, sensitiveness, irritability, sweating, lack of energy, agitation, anxiety, and sleep disturbance. She suffered AE from previous antidepressants and thereby usually treated with subtherapeutic dosages without achievement of adequate response. After switching to bupropion, reduction of core depressive symptoms, improvement of tremulousness, improvement of energy and cognition was observed but not reached a remission (MADRS: 20 to 15, 25.0\% decrease) till 2 months of PGXt.

\section{Case 4}

Mrs. D is a 51-year-old female, a house-wife. Her symptoms included depressed mood, irritability, fluctuation of mood, agitation, severe anxiety, severe sleep disturbance, emptiness, loneliness, and loss of volition. After 2 months treatment with paroxetine, her core depressive symptoms were not properly improved. After switching to fluoxetine and mirtazapine, dramatic improvement of sleep disturbance and reduction of core depressive symptoms were found and remission was achieved at 91 days of PGXt maintaining till today.

\section{Case 5}

Mr. E is a 45-year-old male, working at a business company. He suffered from multiple episodes of suicidal attempt and continuous feeling of suicidal ideation, depressed mood, irritability, inferiority, significant reduction of productivity, sleep disturbance, and hopelessness. Despite of different classes of antidepressant treatments, his depressive symptoms were not improved. After switching to mirtazapine, improvement of sleep and depressive symptoms were noted at day 14 and remission was achieved at 105 days of PGXt maintaining till today. Based on his report, his functional recovery has been the highest over 5 years.

\section{Case 6}

Mrs. F is a 54-year-old female, a gemologist. Her symptoms included severe depressed mood, sadness, lack of interest, social withdrawal, numbness, lack of energy, agitation, anxiety, and sleep disturbance. She had multiple episode of MDD and visited multiple doctors for seeking better treatment. Her core depressive symptoms retained and never remitted since her treatment with multiple antidepressants after onset of MDD. In addition, she was very sensitive to AEs from antidepressants. After switching to escitalopram, reduction of core depressive symptoms was observed with 2 weeks, however, sleep was not improved; mirtazapine was added up and thereafter her sleep was also substantially improved. To augment therapeutic response, methylphenidate was also added. Remission was observed at 93 days after PGXt maintaining till today. Based on her and husband, she never has been as good as now since the onset of MDD.

\section{Case 7}

Mr. G is a 54-year-old male, a health professional. He suffered continuous feeling of loneliness, lack of motivation, lack of energy, and sadness for 1 years; fluoxetine 20 $\mathrm{mg} / \mathrm{d}$ was successful for treatment of his depressive symptoms for a while, however, mild hand tremor was critical AE to him and thus PGX was done. Vortioxetine was selected and it successfully reduced his tremor and remission was also noted at day 49.

\section{Case 8}

Ms. $\mathrm{H}$ is a 23-year-old female, a student. She suffered suicidal ideation, depressed mood, irritability, sleep disturbance, moderate anxiety, decreased productivity, decreased concentration, and lack of volition. Despite of longstanding treatment with sertraline and paroxetine, her depressive symptoms were not significantly improved and also suffered from AEs, headache, hand tremor, worsening of anxiety, and weight gain. After switching to mirtazapine, improvement of sleep and depressive symptoms were noted at day 14, moderate functional recovery was observed at 2 months of PGXt maintaining (MADRS: 21 to $14,33.3 \%$ decrease) aftermath but remission was not achieved. However, her parents reported that she has never been as good as now since the onset of depression.

\section{Case 9}

Mrs. I is a 38-year-old female, a house-wife. She suffered from chronic episode of depression including symptoms of depressed mood, guilty feeling, incompetence as a wife and parents, decreased social activity, sleep disturbance, lack of energy/volition, sensitiveness, and tiredness. Despite of different classes of antidepressant treatments approximately for 3 years, her depressive symptoms were not much improved. After switching to bupropion, mild improvement of anxiety and sleep disturbance were noted at day 
40 but remission was not achieved till at 123 days (MADRS: 30 to 23, 23.3\% decrease). She wanted to find better treatment and thereby she was eventually referred to another hospital with PGXt results as she wanted.

\section{DISCUSSION}

Indeed numerous similar studies have shown the effectiveness and tolerability of PGXt for treatment of TRD patients [4-12], however, the current evidence of PGXt is not yet enough for routine use in daily practice. Although widespread clinical applications of PGXt is still far away from routine practice, available results potentially suggest that PGXt may be a practical tool to guide antidepressant treatment. Hence, the US Food and Drug Administration included PGXt indications in the labeling of several antidepressants [15]. Provisional PGXt guidelines have been also developed and continuously revised to track down the rapid progress of modern PGX study results $[7,16,17]$. The Clinical Pharmacogenetics Implementation Consortium has also continuously published updated-guidelines for relevant information for PGXt [1].

Most current treatment guidelines suggest switching, combination, and augmentation strategies upon a failure of current antidepressant treatment providing inadequate response despite of proper treatment (dose/duration, etc.) in routine practice; however, blind and empirical choice of next antidepressant is also away from systematic and scientific strategy not guaranteeing a successful treatment [18-23]. In addition early treatment response is known to determine the ultimate clinical outcomes, indicating a need of fast and correct treatment decision pathway $[24,25]$. Therefore, PGXt targeting a precision medicine can prompt, facilitate, and widen proper treatment choice for such TRD patients in busy daily practice [24].

In this context our case series with successful PGXt potentially showed its clinical utility and benefit for patients with TRD in routine practice. Sufficient evidence from adequately-powered and well-designed studies should be necessary for clinicians to ensure the utility of PGXt in routine practice.

\section{- Conflicts of Interest}

No potential conflict of interest relevant to this article was reported.

\section{Author Contributions}

Conceptualization: Chi-Un Pae. Protocol development: Chi-Un Pae. Draft writing: Kyung Ho Lee, Chi-Un Pae. Intellectual comments and critics on the content: Alessandro Serretti, Won-Myong Bahk, Soo-Jung Lee. Data acquisition: Chi-Un Pae, Kyung Ho Lee. Data analysis: Chi-Un Pae.

\section{ORCID}

Kyung Ho Lee https://orcid.org/0000-0003-1473-2125 Won-Myong Bahk https://orcid.org/0000-0002-0156-2510 Soo-Jung Lee https://orcid.org/0000-0002-1299-5266 Alessandro Serretti https://orcid.org/0000-0003-4363-3759 Chi-Un Pae https://orcid.org/0000-0003-1632-4248

\section{REFERENCES}

1. Han C, Wang SM, Bahk WM, Lee SJ, Patkar AA, Masand PS, et al. A pharmacogenomic-based antidepressant treatment for patients with major depressive disorder: results from an 8-week, randomized, single-blinded clinical trial. Clin Psychopharmacol Neurosci 2018;16:469-480.

2. Peterson JF, Field JR, Shi Y, Schildcrout JS, Denny JC, McGregor $\mathrm{TL}$, et al. Attitudes of clinicians following large-scale pharmacogenomics implementation. Pharmacogenomics J 2016;16: 393-398.

3. Salm M, Abbate K, Appelbaum P, Ottman R, Chung W, Marder $\mathrm{K}$, et al. Use of genetic tests among neurologists and psychiatrists: knowledge, attitudes, behaviors, and needs for training. J Genet Couns 2014;23:156-163.

4. Pérez V, Salavert A, Espadaler J, Tuson M, Saiz-Ruiz J, SáezNavarro C, et al. Efficacy of prospective pharmacogenetic testing in the treatment of major depressive disorder: results of a randomized, double-blind clinical trial. BMC Psychiatry $2017 ; 17: 250$

5. Espadaler J, Tuson M, Lopez-Ibor JM, Lopez-Ibor F, Lopez-Ibor MI. Pharmacogenetic testing for the guidance of psychiatric treatment: a multicenter retrospective analysis. CNS Spectr 2017;22:315-324.

6. Bradley P, Shiekh M, Mehra V, Vrbicky K, Layle S, Olson MC, et al. Improved efficacy with targeted pharmacogenetic-guided treatment of patients with depression and anxiety: a randomized clinical trial demonstrating clinical utility. J Psychiatr Res 2018;96:100-107.

7. Hall-Flavin DK, Winner JG, Allen JD, Jordan JJ, Nesheim RS, Snyder KA, et al. Using a pharmacogenomic algorithm to guide the treatment of depression. Transl Psychiatry 2012;2: e172.

8. Winner JG, Carhart JM, Altar CA, Allen JD, Dechairo BM. A prospective, randomized, double-blind study assessing the clinical impact of integrated pharmacogenomic testing for 
major depressive disorder. Discov Med 2013;16:219-227.

9. Bousman CA, Müller DJ, Ng CH, Byron K, Berk M, Singh AB. Concordance between actual and pharmacogenetic predicted desvenlafaxine dose needed to achieve remission in major depressive disorder: a 10-week open-label study. Pharmacogenet Genomics 2017;27:1-6.

10. Singh AB. Improved antidepressant remission in major depression via a pharmacokinetic pathway polygene pharmacogenetic report. Clin Psychopharmacol Neurosci 2015;13: 150-156.

11. Hall-Flavin DK, Winner JG, Allen JD, Carhart JM, Proctor B, Snyder KA, et al. Utility of integrated pharmacogenomic testing to support the treatment of major depressive disorder in a psychiatric outpatient setting. Pharmacogenet Genomics 2013;23:535-548.

12. Brennan FX, Gardner KR, Lombard J, Perlis RH, Fava M, Harris $\mathrm{HW}$, et al. A naturalistic study of the effectiveness of pharmacogenetic testing to guide treatment in psychiatric patients with mood and anxiety disorders. Prim Care Companion CNS Disord 2015;17:e1-e7.

13. Rosenblat JD, Lee Y, McIntyre RS. The effect of pharmacogenomic testing on response and remission rates in the acute treatment of major depressive disorder: a meta-analysis. J Affect Disord 2018;241:484-491.

14. Fabbri C, Zohar J, Serretti A. Pharmacogenetic tests to guide drug treatment in depression: comparison of the available testing kits and clinical trials. Prog Neuropsychopharmacol Biol Psychiatry 2018;86:36-44.

15. Fabbri C, Crisafulli C, Calabrò M, Spina E, Serretti A. Progress and prospects in pharmacogenetics of antidepressant drugs. Expert Opin Drug Metab Toxicol 2016;12:1157-1168.

16. Nassan M, Nicholson WT, Elliott MA, Rohrer Vitek CR, Black JL, Frye MA. Pharmacokinetic pharmacogenetic prescribing guidelines for antidepressants: a template for psychiatric precision medicine. Mayo Clin Proc 2016;91:897-907.

17. de Leon J, Armstrong SC, Cozza KL. Clinical guidelines for psychiatrists for the use of pharmacogenetic testing for
CYP450 2D6 and CYP450 2C19. Psychosomatics 2006;47: 75-85.

18. Kennedy SH, Lam RW, Cohen NL, Ravindran AV; CANMAT Depression Work Group. Clinical guidelines for the treatment of depressive disorders. IV. medications and other biological treatments. Can J Psychiatry 2001;46 Suppl 1:38S-58S.

19. Anderson IM, Ferrier IN, Baldwin RC, Cowen PJ, Howard L, Lewis $\mathrm{G}$, et al. Evidence-based guidelines for treating depressive disorders with antidepressants: a revision of the 2000 British Association for Psychopharmacology guidelines. J Psychopharmacol 2008;22:343-396.

20. Bauer M, Pfennig A, Severus E, Whybrow PC, Angst J, Möller $\mathrm{HJ}$; World Federation of Societies of Biological Psychiatry. World Federation of Societies of Biological Psychiatry (WFSBP) Guidelines for Biological Treatment of Unipolar Depressive Disorders, part 1: update 2013 on the acute and continuation treatment of unipolar depressive disorders. World J Biol Psychiatry 2013;14:334-385.

21. Patkar AA, Pae CU. Atypical antipsychotic augmentation strategies in the context of guideline-based care for the treatment of major depressive disorder. CNS Drugs 2013;27 Suppl 1:S29-S37.

22. Wang SM, Han C, Pae CU. Criticisms of drugs in early development for the treatment of depression: what can be improved? Expert Opin Investig Drugs 2015;24:445-453.

23. Wang HR, Bahk WM, Seo JS, Woo YS, Park YM, Jeong JH, et al. Korean Medication Algorithm for Depressive Disorder: comparisons with other treatment guidelines. Clin Psychopharmacol Neurosci 2017;15:199-209.

24. Serretti A. The present and future of precision medicine in psychiatry: focus on clinical psychopharmacology of antidepressants. Clin Psychopharmacol Neurosci 2018;16:1-6.

25. Han C, Wang SM, Bahk WM, Lee SJ, Patkar AA, Masand PS, et al. The potential utility of aripiprazole augmentation for major depressive disorder with mixed features specifier: a retrospective study. Clin Psychopharmacol Neurosci 2019;17: 495-502. 